\title{
The effects of ambient impurities on the surface tension
}

\author{
A. Ponce-Torres ${ }^{1, a}$ and E. J. Vega ${ }^{1, b}$ \\ ${ }^{1}$ Depto. de Ingeniería Mecánica, Energética y de los Materiales, \\ Universidad de Extremadura, E-06006 Badajoz, Spain
}

\begin{abstract}
A liquid bridge is a liquid column held captive between two coaxial and parallel solid disks. It is an excellent test bench where measuring the surface tension. In this paper, we used this fluid configuration to examine experimentally the effects of ambient impurities on the surface tension over time. For this purpose, the liquid bridge equilibrium shape was analyzed when the liquid bridge was surrounded by three environments: the uncontrolled ambient, and both air and argon encapsulated in a small glass cover. Ambient contamination produced a sharp decrease of the surface tension of ultra-pure water. The presence of an anionic surfactant in the free surface of an aqueous solution did not inhibit the action of impurities coming from the ambient. Impurities can influence the dynamical behavior of the free surface in flows dominated by the surface tension. Therefore, a careful control of that influence can be crucial in many applications of fluid mechanics.
\end{abstract}

\section{Introduction}

What role plays the ambient impurities in the surface tension of liquids? The usual answer is: it can be regarded as negligible for the first minutes of a certain experiment involving exposed liquids to an uncontrolled ambient. It is believed that liquids with low surface tensions $(20 \lesssim \sigma \lesssim$ $30 \mathrm{mN} / \mathrm{m}$ ) are almost insensitive to impurities. Because of the fact that the surface tension of the pure liquid is small, impurities of surfactant nature cannot lower significantly that tension. However, impurities can affect noticeably the behavior of capillary systems with high surface tensions. For example, they can cause Marangoni convection due to the gradients of surface tension over the interface. This phenomenon is particularly important in experiments with air-water interfaces.

The presence of a tainted monolayer due to the ambient impurities could produce an extra-dissipation of energy in millimetric liquid free surface [1]. In fact, surface contamination is known to play a significant role in surface wave damping [2] (and in surface wave dynamics too, see Refs. [3] and [4] when water is used). The measurement of surface tension over time can help us to know whether ambient impurities are forming a monolayer on the free surface (even if surfactants are previously added on purpose) which could affect our experimental results.

Liquid bridges are excellent test benches where analyzing varied phenomena associated with surface tension [5-8]. The large surface/volume ratios of millimeter bridges make the surface tension force dominant in most situations. Liquid bridges offer significant advantages over their close relatives: the pendant and sessile drops. When

\footnotetext{
a e-mail: albertoponce0692@gmail.com

be-mail: ejvega@unex.es
}

working with liquid bridges, the experimenter has at his disposal an additional adjustable parameter, the slenderness, to enlarge the range of experimental conditions. In addition, the liquid bridge shape remains sensitive to the surface tension value even in the minimum volume stability limit, while drops become spherical independently of the surface tension value for small enough volumes.

The liquid bridge configuration considered in this work (figure 1) consists of an isothermal mass of liquid of volume $\mathcal{V}$, held between two parallel coaxial circular solid supports placed a distance $L$ apart. The radius of the two solid supports is $R$. Due to the sharpness of their edges, one assumes that the liquid anchors perfectly to those edges, preventing motion of the triple contact line. Hence, spreading of the liquid over the lateral surfaces of the solid supports does not occur. The liquid bridge is surrounded by another fluid medium of negligible density and viscosity, and is subjected to the action of the force of gravity of magnitude $g$ per unit mass. The liquid bridge density and viscosity are $\rho$ and $\mu$, respectively, while $\sigma$ is the surface tension associated with the interface. For this axisymmetric configuration, the equilibrium contour of the liquid bridge is characterized by the function $F_{0}(z)$, which measures the distance between a surface element and the axis of the disks ( $z$ axis). The dimensionless parameters characterizing the fluid configuration are the slenderness $\Lambda \equiv L /(2 R)$, the reduced volume $V \equiv \mathcal{V} /\left(\pi R^{2} L\right)$, the static Bond number $B_{0} \equiv \rho g R^{2} / \sigma$, and the Capillary number (defined as the square root of the Ohnesorge number) $C_{\mu} \equiv \mu(\rho \sigma R)^{-1 / 2}$

In this work, we will examine experimentally the effects of ambient impurities on the surface tension over time. For this purpose, the liquid bridge equilibrium shape will be analyzed when the liquid bridge is surrounded by 


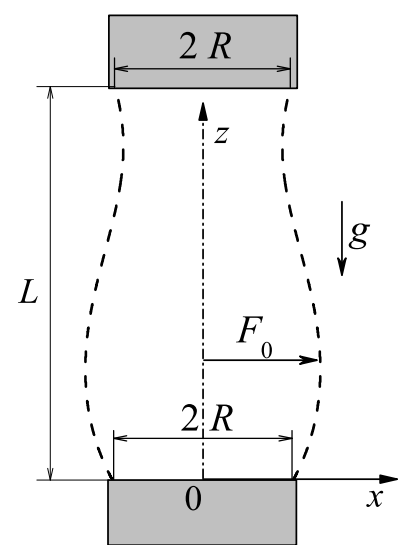

Figure 1. Liquid bridge configuration.

three environments: the uncontrolled ambient, and both air and argon encapsulated in a small glass cover. We will analyze the effects of impurities in both pure water and an aqueous solution of an anionic surfactant. The paper is organized as follows. The experimental method used to analyze the problem is described in Sec. 2. The results are presented and discussed in Sec. 3. Finally, the paper closes with some concluding remarks in Sec. 4.

\section{Experimental Method}

Figure 2 shows the experimental setup used in the present study. A liquid bridge was formed between an upper needle (A) and a coaxial bottom disk (B), both about $1 \mathrm{~mm}$ in radius and placed in a cubic cell $(\mathrm{C})$. The upper needle could be displaced along its axis, and was used to feed and remove liquid by using a syringe pump connected to a stepping motor. The cell was mounted on a platform (D). Digital images of $704 \times 600$ pixels were acquired using a CMOS camera (E), equipped with a set of optical lenses $(\mathrm{F})$ providing a frame covering an area of about $4 \times 3.4 \mathrm{~mm}$. The magnification obtained was 5.73 $\mu \mathrm{m} /$ pixel. The camera could be displaced both horizontally and vertically using a triaxial translation stage $(G)$ to focus the liquid bridge. The fluid configuration was illuminated from the back side by cool white light provided by an optical fiber $(\mathrm{H})$ connected to a light source. A frosted diffuser (I) was positioned between the optical fiber and the cell to provide a uniformly lit background. All these elements were mounted on an optical table with a pneumatic anti-vibration isolation system ( $J$ ) to damp the vibrations coming from the building. The experiments were performed at room temperature, approximately $T=25 \pm 1$ ${ }^{\circ} \mathrm{C}$. The images of the liquid bridge at equilibrium were processed with the Theoretical Image Fitting AnalysisAxisymmetric Interfaces (TIFA-AI) method [9] to measure the surface tension.

The test liquids used in the experiments were deionized water for synthesis (Di-water, Merck) $(\rho=998$ $\mathrm{kg} / \mathrm{m}^{3}$, and $\mu=0.001 \mathrm{~kg} / \mathrm{ms}$ ), and an aqueous solution of the anionic surfactant SDS (sodium dodecyl sulfate for

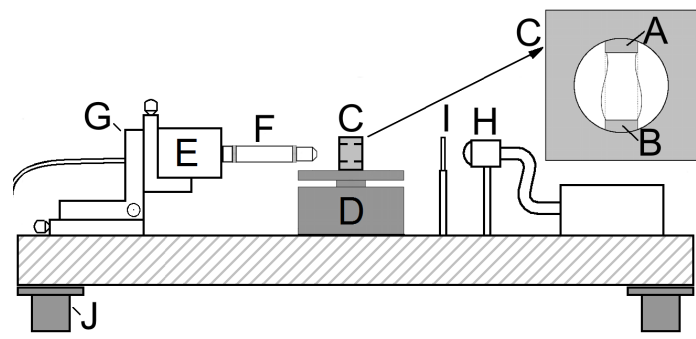

Figure 2. Experimental apparatus: upper needle (A), bottom disk (B), liquid bridge cell (C), platform (D), camera (E), optical lenses $(F)$, micrometer screws $(G)$, optical fiber $(H)$, frosted diffuser (I), and optical table (J).

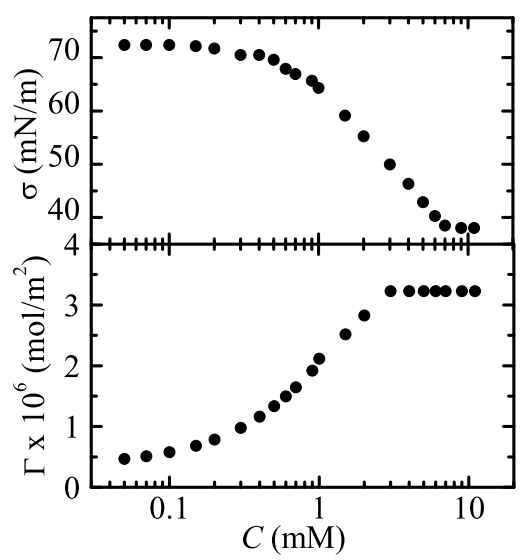

Figure 3. Surface tension and surface density for aqueous SDS solutions as a function of the bulk surfactant concentration $C$. The points come from the interpolation of the data in the reference [10].

molecular biology, assay $=99.0 \%$ (GC, gas chromatography), Sigma Aldrich). The concentration was $C=0.84$ $\mathrm{mM}$, much smaller than the accepted value $8 \mathrm{mM}$ of the cmc (critical micelle concentration), and than that leading to the maximum package surface density of aqueous solution of SDS, around $3 \mathrm{mM}$. In figure 3, one can observe the values of surface tension and surfactant surface density $\Gamma$ for aqueous SDS solutions as a function of the bulk surfactant concentration $C$. The points come from the interpolation of the data in the reference [10], where is mentioned that both surfactant (SDS) and water were carefully purified in order to minimize the effects of impurities. Therefore, these curves can be interpreted as "ideal" curves for aqueous SDS solutions.

We conducted experimental runs with both test liquids, Di-water and $0.84 \mathrm{mM}$ solution of SDS in Di-water, for three different environments: the uncontrolled ambient of air, and saturated air and argon (ultra-pure argon, purity 5.0 , Linde, $\rho=1.7 \mathrm{~kg} / \mathrm{m}^{3}$ ) encapsulated in a small glass cover, repeating each experimental run to check the reproducibility of the results. Argon was chosen because it is the least active gas with liquids, water-insoluble, and its density is higher than air, which makes easier create the argon ambient in the cover. Figure 4 shows the small glass cover introduced in the liquid bridge cell of the experi- 


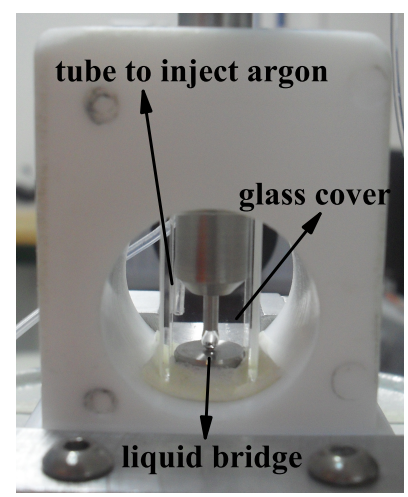

Figure 4. Image of the liquid bridge cell with a glass cover to control the ambient.

mental setup to create the controlled ambients. The optical glass cover was closed hermetically, only in the bottom part, thanks to a rubber gasket. In the case of controlled ambient of air, the bottom part of the glass cover was filled with a small amount of Di-water to saturate the ambient, while argon was injected through a small plastic tube placed in the glass cover (as it can be seen in the figure) to obtain a ambient of argon. The velocity of the argon flow was low enough to neglect any effects on the liquid bridge.

In each experimental run: a liquid bridge was formed, and one image of the liquid bridge was acquired approximately every 2 minutes. We measured the surface tension over time in the first 23 minutes after forming the liquid bridge. During the experiments, the liquid bridge volume was reduced over time due to the evaporation (see figure 5 ), with the exception of the cases in which the liquid bridge was encapsulated in saturated air. For the experiments of $0.84 \mathrm{mM}$ aqueous SDS solution, it was supposed that the evaporation did not change the concentration because the diffussion of the surfactant particles from the needle to the bulk/free surface of the liquid bridge can be regarded as immediate if it is compared with the evaporation rate. In other words, there is always a spices and thermodynamic equilibrium between the injection system and the bulk of the liquid bridge. Consequently, the bulk concentration and its associated surface density remains constant even when the volume of the liquid bridge changes. All the experiments were conducted with extreme care to avoid the contamination of the liquid during its injection and the formation of the liquid bridge.

\section{Results}

Figure 6 shows the results obtained for the interface contamination over time for Di-water. As can be observed, surface tension depends strongly on time in all the cases. In our experiments, only when a controlled ambient of saturated air or argon is used, it is possible to get a value next to the well-known value of $72 \mathrm{mN} / \mathrm{m}$ for pure water, over the first 5 minutes. When the liquid bridge is surrounded by an uncontrolled ambient the value of surface tension is

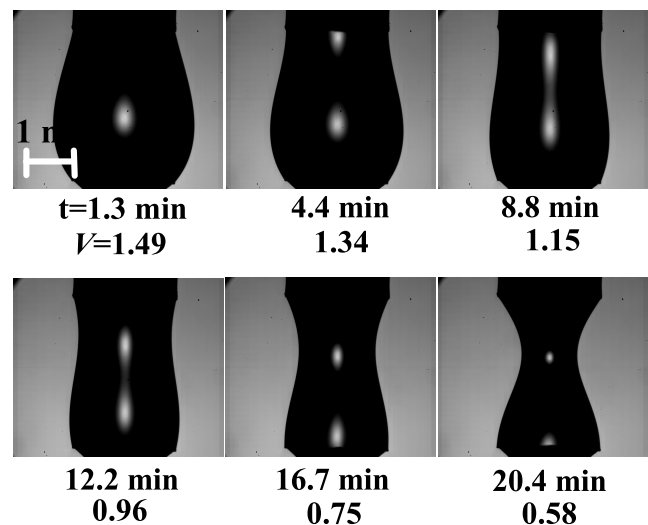

Figure 5. Images of the liquid bridge captured during the experimental run of the $0.84 \mathrm{mM}$ aqueous SDS solution in the argon environment.

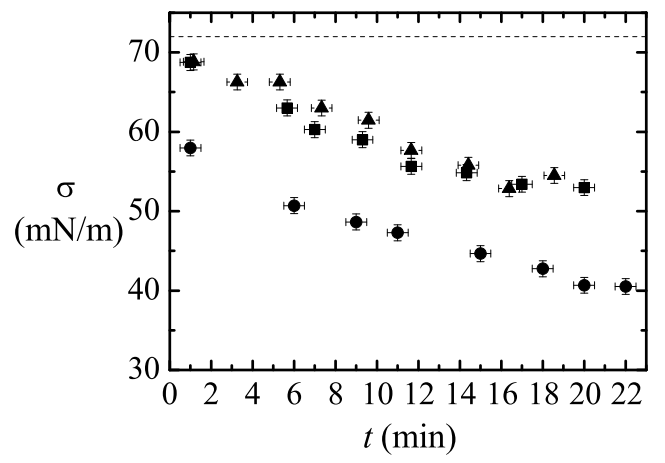

Figure 6. Surface tension as a function of the time $t$ when a liquid bridge of Di-water is surrounded by three environments: uncontrolled ambient (solid circles), saturated air (solid squares) and argon (solid triangles). The dashed line indicates the ideal value of surface tension for Di-water, $72 \mathrm{mN} / \mathrm{m}$. The parameters characterizing the experiments were $\Lambda=1.45, B_{0}=0.135-0.25$, and $C_{\mu}=3.73-5 \times 10^{-3}$.

already under $60 \mathrm{mN} / \mathrm{m}$ in the first minute of our experimental run, indicating that the impurities reach rapidly the liquid free surface in that period. Thus a controlled ambient slows down that first quick contamination. After 20 minutes exposed to the uncontrolled ambient the surface tension goes down to $40 \mathrm{mN} / \mathrm{m}$ (from $58 \mathrm{mN} / \mathrm{m}$ ). In both saturated air and argon ambients encapsulated in a small glass cover, the surface tension reachs an almost constant value around $53 \mathrm{mN} / \mathrm{m}$ after 15 minutes.

Figure 7 allows one to analyze the effects of impurities in an aqueous solution of SDS surfactant. In this figure, we plot the results for a $0.84 \mathrm{mM}$ aqueous SDS solution exposed over time at the same three environments used with the previous liquid. Interestingly, although the free surface of the liquid bridge is doped with a surfactant, the latter does not inhibit the action of impurities coming from the ambient. When surfactant is introduced in the problem, it can be seen only a slight difference between the values of surface tension for the different environments in the first minute, since the free surface is previously doped with the 


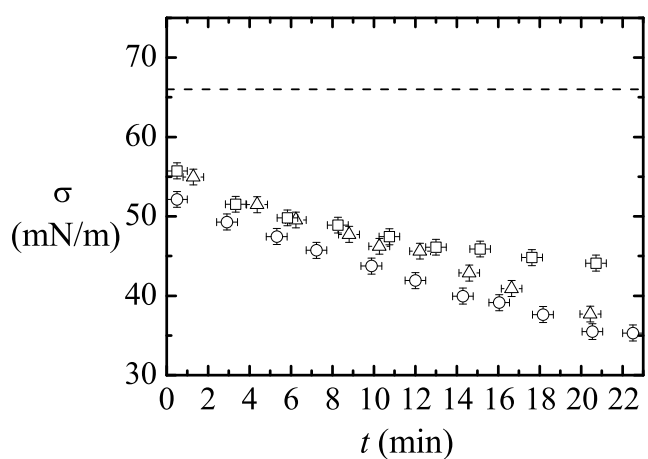

Figure 7. Surface tension as a function of the time $t$ when a liquid bridge of $0.84 \mathrm{mM}$ aqueous SDS solution is surrounded by three environments: uncontrolled ambient (open circles), saturated air (open squares) and argon (open triangles). The dashed line indicates the ideal value of surface tension for a $0.84 \mathrm{mM}$ aqueous SDS solution, around $66 \mathrm{mN} / \mathrm{m}$ from the figure 3 . The parameters characterizing the experiments were $\Lambda=1.45, B_{0}=$ $0.175-0.28$, and $C_{\mu}=4.23-5.35 \times 10^{-3}$.

surfactant molecules. The discrepancy between the ideal value $66 \mathrm{mN} / \mathrm{m}$ (dashed line in the figure) and our results in the first minute may be due to a lack of purificaction of the components of our solution [10]. Since surfactants are by definition highly surface active, chemical impurities in the solution often tend to be surface active and may affect surface measurements. In the uncontrolled ambient, the surface tension suffers an important reduction after 20 minutes in the experiment (from 52 to $35 \mathrm{mN} / \mathrm{m}$ ). On contrary, in the saturated air ambient the surface tension decreases more slowly, and after 15 minutes a transition to a constant value around $44 \mathrm{mN} / \mathrm{m}$ can be obseved. Finally, when argon is used as environment the surface tension changes sharply, as it happens in an uncontrolled ambient. However, the values of surface tension are above of the uncontrolled ambient case.

\section{Conclusions}

The effects of ambient impurities on the surface tension over time was analyzed experimentally. The change in the surface tesion for water and an aqueous solution of a surfactant over time was relevant in all the cases. Then, the presence of a surfactant on the free surface did not inhibit the action of impurities coming from the ambient. In general, the use of a controlled ambient allowed us to keep the surface tension around values higher than the uncontrolled case, and after around 15 minutes it seemed to reach a constant value.

Thus, in this work it was shown impurities can influence the dynamical behavior of the free surface in flows dominated by the surface tension. A careful control of that influence can be crucial in many applications of fluid mechanics. For this porpuse, an accurate knowledge of the surface tension value over time is crucial to properly analyze the experimental results.

\section{References}

[1] M.A. Herrada, J.M. Montanero, J.M. Vega, Phys. Fluids 23, 082102 (2011)

[2] J.A. Nicolás, J.M. Vega, J. Fluid Mech. 410, 367 (2000)

[3] D.M. Henderson, J. Fluid Mech. 365, 89 (1998)

[4] E. Martín, J.M. Vega, J. Fluid Mech. 546, 203 (2006)

[5] J. Eggers, Rev. Mod. Phys. 69, 865 (1997)

[6] Q.S. Chen, W.R. Hu, Int. J. Heat Mass Transfer 41, 825 (1998)

[7] J.C. Burton, J.E. Rutledge, P. Taborek, Phys. Rev. Lett. 92, 244505 (2004)

[8] E.J. Vega, J.M. Montanero, C. Ferrera, M.A. Herrada, Meas. Sci. Technol. 25, 0753031 (2014)

[9] M.G. Cabezas, A. Bateni, J.M. Montanero, A.W. Neumann, Colloids Surf. A 255, 193 (2005)

[10] A.J. Prosser, E.I. Franses, Colloid Surf. APhysicochem. Eng. Asp. 178, 1 (2001) 